\title{
Neuro-Fuzzy Based ECG Signal Classification with A Gaussian Derivative Filter
}

\author{
J. M. Abdul-Jabbar* S. N. M. Al-Faydi** and H. N. Yahya** \\ * Computer Eng. Dept., College of Eng., University of Mosul, Mosul, Iraq. \\ ** Computer and Information Eng. Dept., College of Electronic Eng., University of Mosul, Mosul, Iraq.
}

\begin{abstract}
In this paper, a neuro-fuzzy classification method is used for identifications of ECG signals. A feature extraction method with a QRS like filter (first order Gaussian derivative filter) is used. Five standard parameters (energy, mean value, standard deviation, maximum and minimum) are extracted from these disease features and then used as inputs for the neuro-fuzzy classification system. The ECG signals are imported from the standard MIT-BIH database. Five types of ECG signals are used for classification; they are normal sinus rhythm (NSR), left bundle branch block (LBBB), right bundle branch block (RBBB), premature ventricular contraction (PVC) and pacemaker (PM). The proposed system combines the neural network adaptive capabilities and fuzzy inference system with the suitable filter design to give a promising classification accuracy of $99 \%$.
\end{abstract}

Keywords: Neuro-fuzzy system, ECG signal, Gaussian Derivative Filter, QRS-like filter, NSR, LBBB, RBBB, PVC and PM.

$$
\begin{aligned}
& \text { تصنيف اشارة القلب باعتماد النظام العصبي المضبب ومرشح نوع مشتقة كاوس }
\end{aligned}
$$

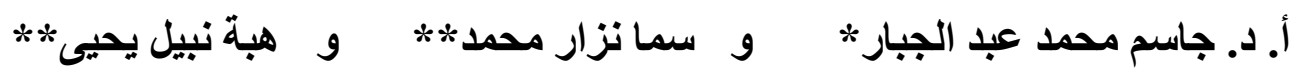

$$
\begin{aligned}
& \text { * قسم هندسة الحاسوبـ كلية الهندسةـ جامعة الموصل- الموصل- العراق. }
\end{aligned}
$$

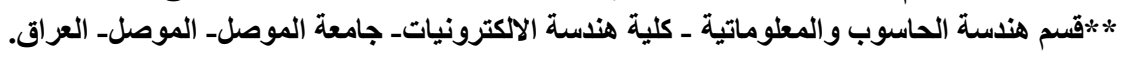

\section{الخلاصة}

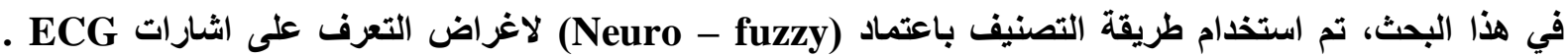

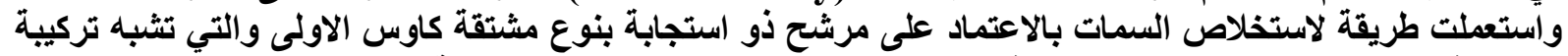

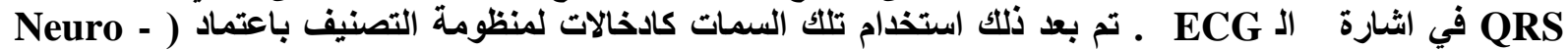

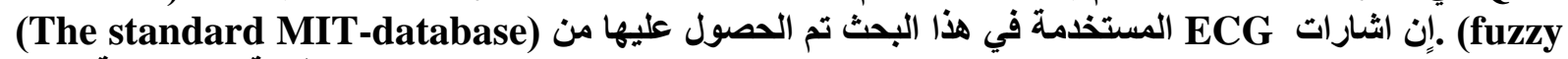

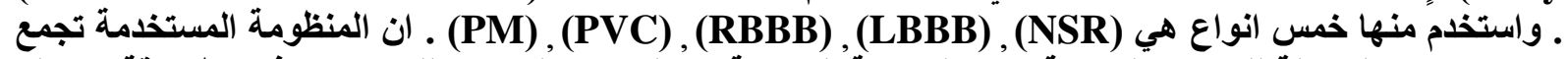

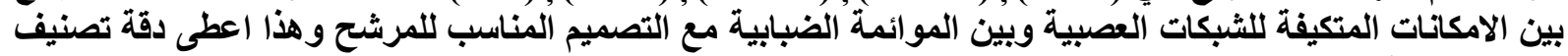
واعدة وينسبة 999\% م الأنات 


\section{INTRODUCTION}

In recent years, classification of ECG signals has received great interest from the biomedical engineering community. The ECG signal is a measurement of the electrical activity from the heart muscles. Intercardiac signals, generated by the action potentials of different cardiac parts, pass through various body layers, and can be finally picked up as an ECG signal through electrodes attached to some points on the skin surface [1]. Usually, ECG meter converts the heart electrical activity into graphs on paper as shown in Fig. 1.

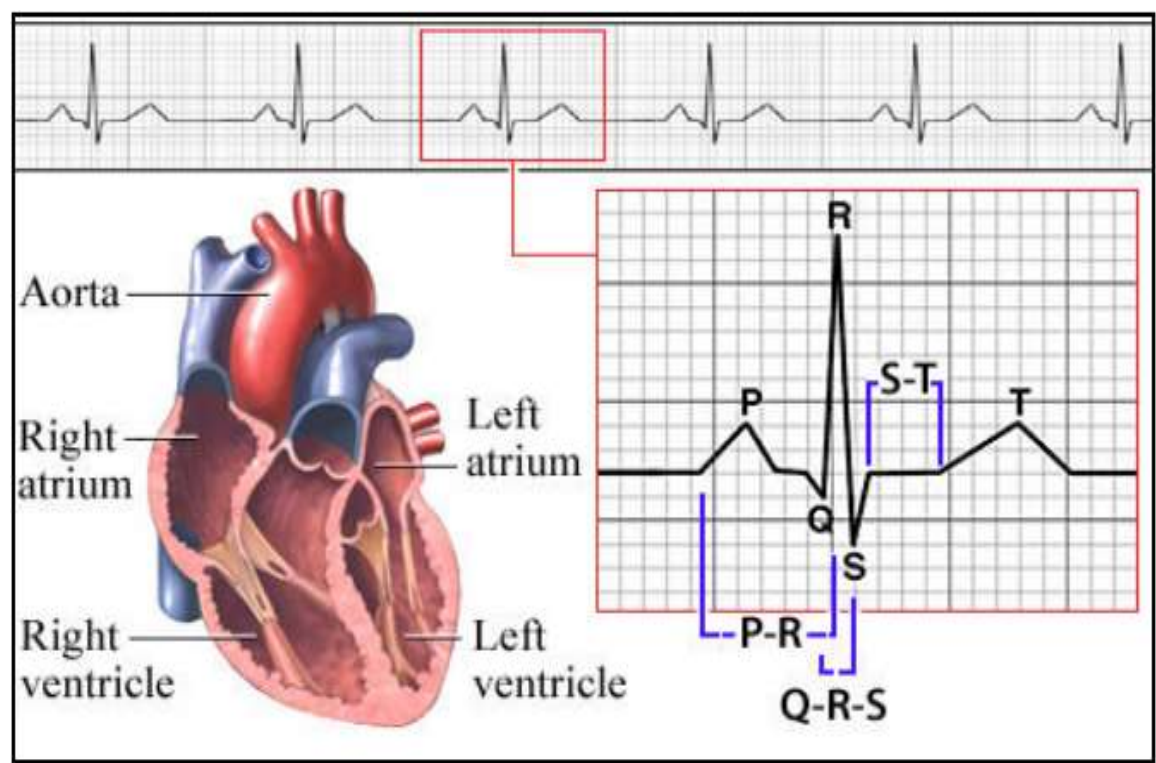

Fig. 1 Diagram of the human heart and example of normal ECG trace.

The most important part in ECG signal components is the QRS complex that is associated with electrical ventricular activation. ECG pattern recognition consists of a sequence of stages; starting with a feature extraction stage to extract features from the occurring patterns. This stage converts the patterns to features that are regarded as a condensed and less correlated representation of the ECG signal [2].

The extracted features from ECG signal play a vital role in diagnosing the cardiac disease. The purpose of feature extraction is to find some properties within the non-stationary ECG signal that would allow successful abnormality detection and efficient prognosis [3]. Several techniques have been used for classification of ECG signals, such as digital signal analysis, artificial neural network, fuzzy logic methods, hidden Markov model, genetic algorithm, support vector machines and Bayesian method [4].

One of the most popular QRS detection algorithms was proposed by Pan and Tomkins in 1985 [5]. Such QRS detection algorithm works in the time domain based on analysis of the slope, amplitude and width of the QRS complex (referring to the depolarization of the right and left ventricles). Fig. 2 shows a block diagram of the Pan-Tompkins algorithm. In Fig. 2, the band-pass filter $(5 \mathrm{~Hz}-17 \mathrm{~Hz})$ is composed of a low-pass filter and a high-pass filter. The signal is then differentiated. The derivative operator suppresses the low-frequency elements ( $\mathrm{P}$ and $\mathrm{T}$ waves) and picks the high frequencies out (information about the QRS complex slope). For easier QRS complex interpretation, squaring operation is used in the next step to 
make all values positive. After the squaring operation, multiple peaks are observed within the QRS complex. To eliminate this, the movingwindow integration filter is being performed. To detect QRS complexes an adaptive thresholding is applied. New peak is marked when a local maximum is found during the defined period [5].

In 2008, B. Anuradha, et al [6] used an adaptive neuro-fuzzy inference system (ANFIS) classifier in a complex investigating algorithms.

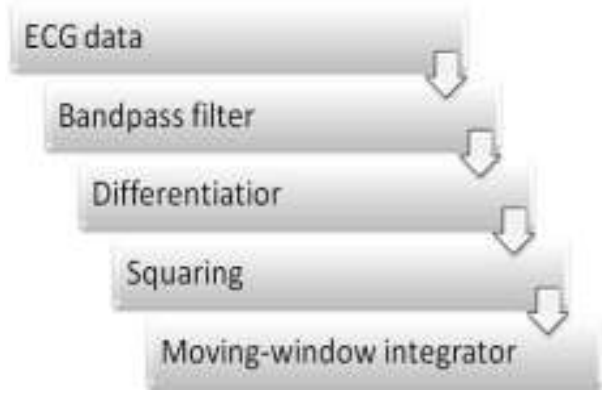

Fig. 2 The Pan-Tompkins algorithm Compared to analytical method, very interesting recognition and classification capabilities were obtained and an accuracy rate of $94 \%$ was achieved.

In 2010, T. M. Nazmy, et al [7] proposed a method for ECG beat classification. Such method was consisted of the following steps: independent component analysis (ICA), power spectrum to extract important feature together with the RR time interval between the R points of the present and previous beats and ANFIS classifiers. Six types of ECG samples were selected from the MIT-BIH arrhythmia database for experiments. A high level of accuracy of more than $97 \%$ was achieved.

Also in 2010, N. H. Kamarudin [8] presented the classification of ECG signals to detect arrhythmia and ischemia diseases. Features after discrete wavelet decomposition (DWT) were used as inputs to a neuro-fuzzy classifier. For experimental purposes, ECG samples were selected from three different database sets including the MIT-BIH database. High levels of accuracies (less than 98\%) were achieved.

In 2011, A. Sharma and T. Sharma [9] proposed a technique for heart beat recognition. The individual beats belonging to different categories from the MIT-BIH arrhythmia database were extracted using an R-peak detection algorithm. Features were extracted then from the beats using Principal Component Analysis (PCA). Feature vectors with reduceddimensionality were obtained. Those feature vectors were used to train a neural network (NN) classifier. A separate dataset (not used during training) was tested for performance evaluation. High values of sensitivity and accuracy were also obtained.

In this paper, the two steps of band pass filter and differentiator of Fig. 2 are replaced by some baseline adjustment with noise removing under preprocessing operations and a QRSlike filter (the first order Gaussian derivative filter) in order to extract feature extraction form the ECG signal. Other steps of the algorithm of Fig. 2 ( such as squaring and moving window integrator) are replaced by a neuro-fuzzy-based classification system.

This paper presents the first order Gaussian derivative filter based approach to extract features from ECG signals. A fuzzy-hybrid neural network is developed to recognize and classify five different types of ECG signals. Such neuro-fuzzy method is composed of two sub-networks connected in cascade: the fuzzy self-organizing layer performing the pre-

classification task and the following layers perceptron working as the final classifier. The final classifier classifies the applied input ECG signal to its appropriate class.

This paper is organized as follows: in addition to this introductory section, section II section IV, the results and discussions are given. Finally, section V concludes this paper.

\section{ECG SIGNAL DISCRIPTION}

The tested ECG signals were downloaded and recorded from the PhysioNet database using MIT-BIH Arrhythmia database which is generally recognized as a standard test bench for the evaluation of arrhythmia detectors. All samples are at a sampling frequency of $360 \mathrm{~Hz}$ and one 
lead must be chose to extract the ECG signal, the lead that had been chosen in the proposed work was lead II, so most of the rhythms are seen in this lead record. This paper focuses on four types of cardiac arrhythmias for classification, which are premature ventricular contraction (PVC). The PVC is defined by premature occurrence of a QRS complex which is strange in shape and has duration usually greater than normal QRS complex. For clinical diagnostic, the PVC arrhythmia is most important to be detected [9].

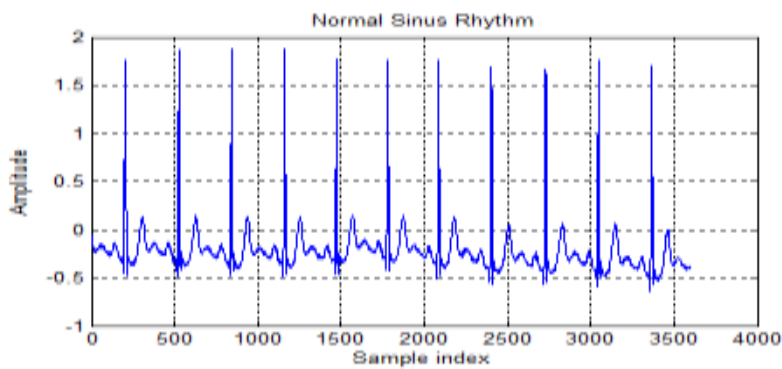

(a)

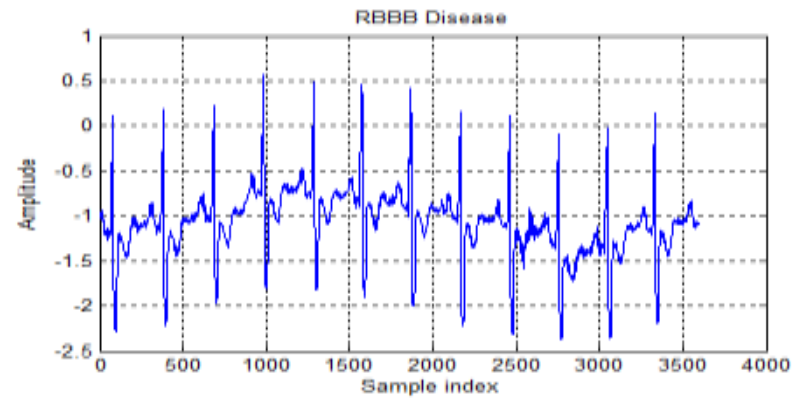

(c)

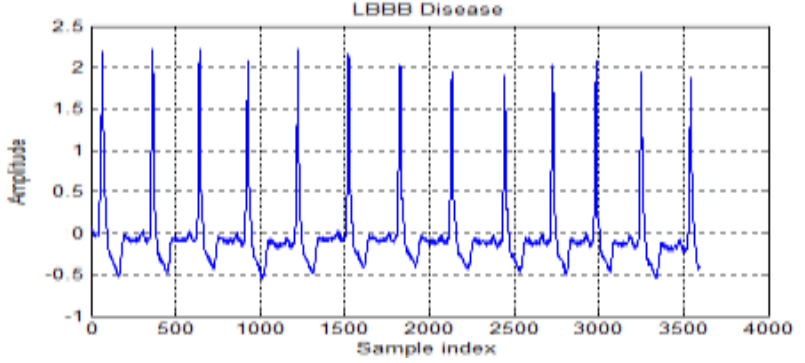

(b)

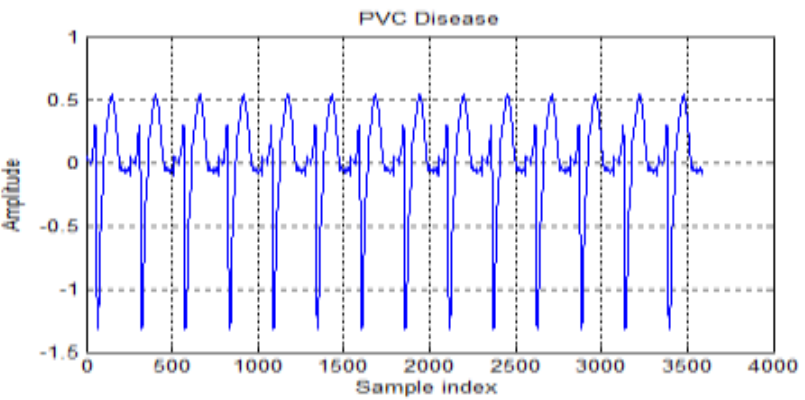

(d)

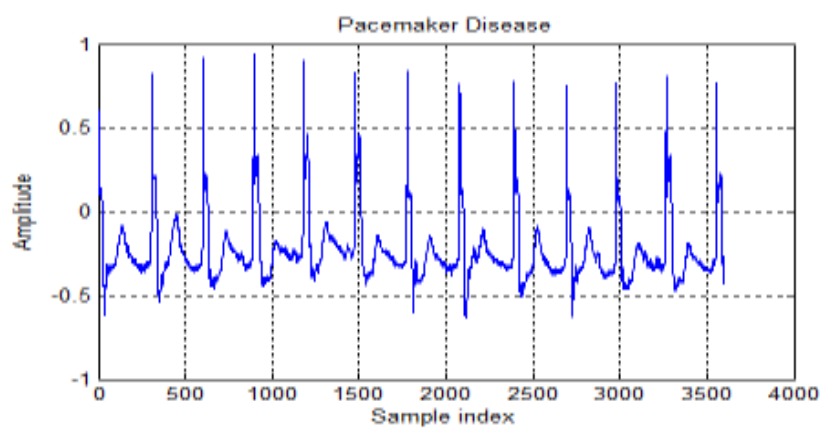

(e)

Fig. 3 Five classes of ECG signals; (a) Normal Sinus Rhythm; (b) Left Bundle Branch Block beats; (c) Right Bundle Branch Block beats; (d) Premature Ventricular Contraction beats; (e) Pacemaker beats.

The second type of cardiac arrhythmias is bundle branch block that belongs to a group of heart diseases called Intra-ventricular conduction defects (IVCD). Bundle branches consist of right and left. The right bundle carries nerve impulses that cause contraction of the right ventricle (the lower chamber of the heart) and happens less often from underlying heart diseases. The left bundle carries nerve impulses that cause contraction of the left ventricle and 
usually happens as a consequence of other diseases such as arteriosclerosis, congenital heart disease, rheumatic fever, myocardial infarction and myocarditis [10]. The last type of cardiac arrhythmias is pacemaker (PM) which responsible for generating initial beat. Therefore, any problem in the pacemaker will make the $\mathrm{P}$ wave disappear. The rest of ECG signal will be generated in atriaventrical (AV) node. For a solution to this problem, electrodes can be placed in the right ventricle (usually at the apex) as pulse generator implanted under the skin [11]. The ECG signals belong to the tested five classes are shown in Fig. 3.

\section{THE PROPOSED METHOD}

The block diagram of the proposed method for ECG signal classification is shown in Fig. 4. The method is divided into three steps: (A) preprocessing (B) feature extraction and (C) classification by Neuro-Fuzzy system.

\section{A. Pre-processing of ECG Signals}

This stage includes five levels of ECG signal pre-processing: in the first level, the ECG signal is normalized. The normalization is necessary to reshape all features to the same base level. Then in the second level, the mean value is removed from each normalized ECG signal. Since the baseline of the ECG signal may be shifted from zero line due to patient movements, it has to be adjusted at zero line for more time enhancement [7]. The fourth stage is employed to remove the noise from ECG signal using Low pass and High pass filters.

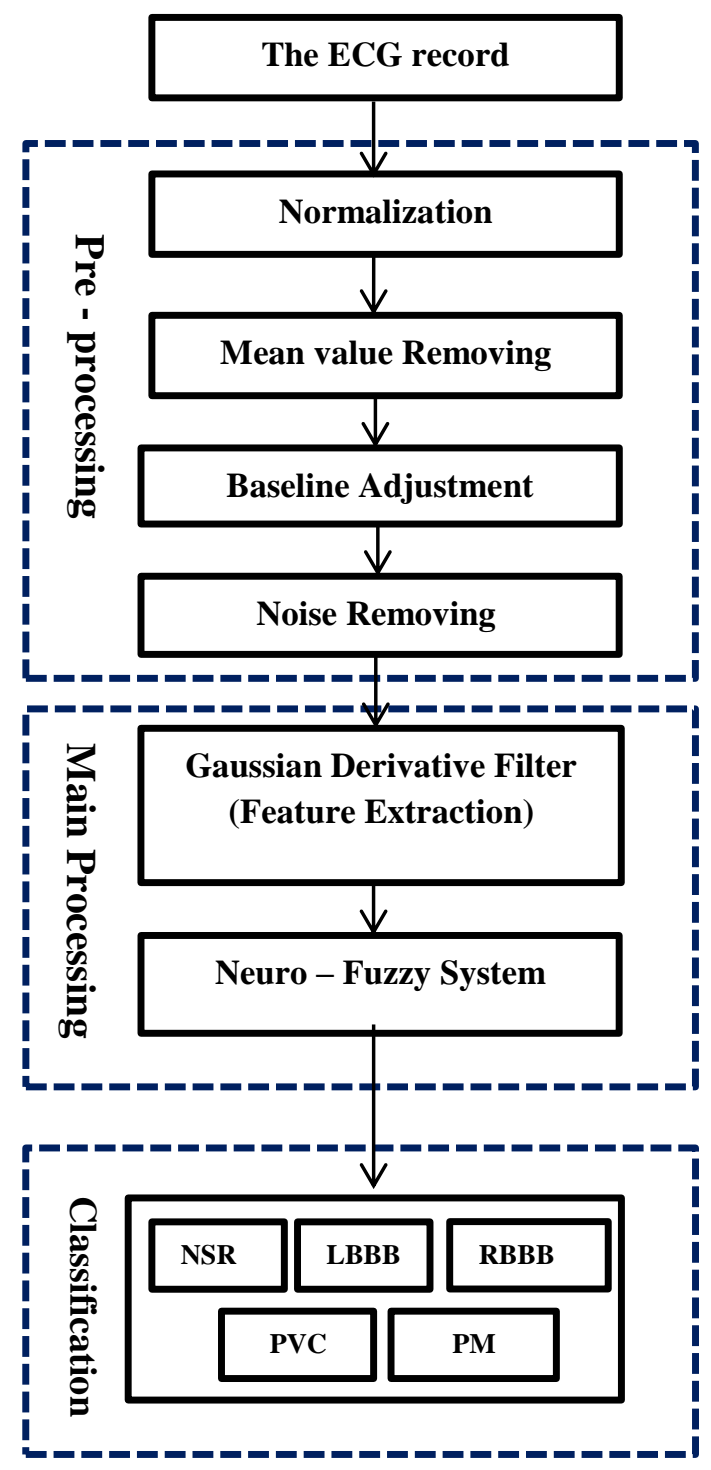

Fig. 4 Block diagram of the proposed method.

\section{B. Feature Extraction}

Features extraction stage includes extracting and transforming the input ECG signal information into a set of features that called feature vector, thereby reducing the data representation pattern. The features set will extract the information from the input ECG signal in order to perform the classification part. The transform of ECG signal is another form of representing the same signal. It does not change the information content present in the ECG signal [8].

In this paper, the first order Gaussian derivative filter is used to extract the features of the ECG signal. The equation of this filter is given by [12] 
$g^{\prime}(t ; \sigma)=\frac{m-t}{\sigma^{3} \sqrt{2 \pi}} e^{\frac{-(t-m)^{2}}{2 \sigma^{2}}}$

Since the first order Gaussian derivative function has a shape that is similar to QRS complex part of the ECG signal, so it can possess the best-fit for QRS feature extraction. The Gaussian derivative function is approximately zero for $|t|>4 \sigma$ where $g^{\prime}(t ; \sigma)<0.0004$ for $|t|$ $>4 \sigma[12]$. The standard deviation $\sigma$ that is proposed in this paper is equal to 4 in order to obtain a filter with approximately similar impulse response shape to the QRS part of the ECG signal. In this case, impulse response of the filter will contain 33 coefficients with the following system function that corresponds to the sampled version given in Fig 5.

$H(z)=\sum_{n=0}^{32} h_{n} z^{-n}$

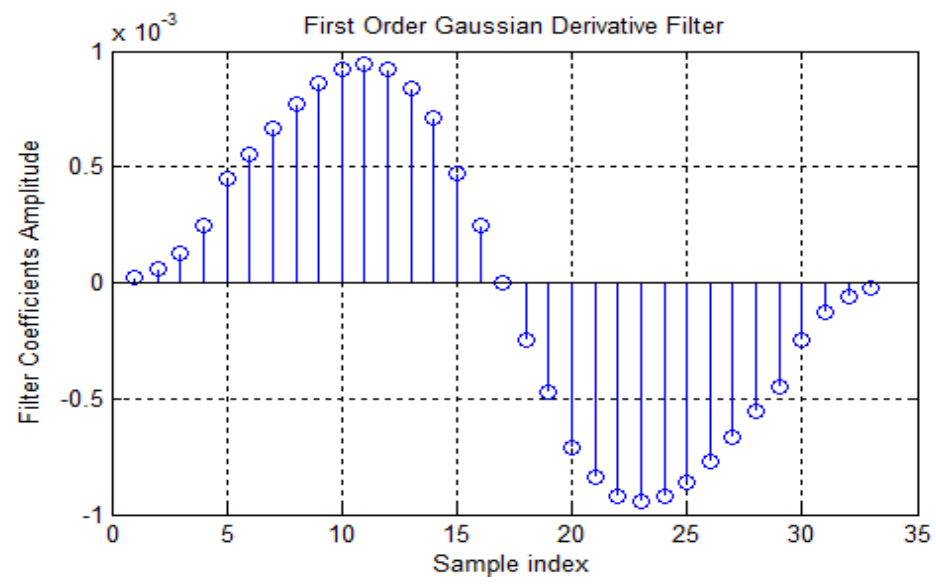

Fig. 5 Impulse response of the $1^{\text {st }}$ order Gaussian derivative filter with $\sigma=4$.

Using polyphase FIR representations, equation (2) can be expressed as

$$
H(z)=H_{\text {even }}\left(z^{2}\right)+z^{-1} H_{\text {odd }}\left(z^{2}\right)
$$

where

$$
\begin{aligned}
& H_{\text {even }}\left(z^{2}\right)=\sum_{n=0}^{16} h_{2 n} z^{-2 n} \\
& H_{\text {odd }}\left(z^{2}\right)=\sum_{n=0}^{15} h_{2 n+1} z^{-2 n}
\end{aligned}
$$

the forms of equations (4) and (5) can be rewritten after down-sampling in terms of $\mathrm{z}$, as

$H_{\text {even }}(z)=\sum_{n=0}^{16} h_{2 n} z^{-n}$

and

$H_{\text {odd }}(z)=\sum_{n=0}^{15} h_{2 n+1} z^{-n}$ 
Figure 6 represents the realization of two down-sampling branches polyphase transfer function of the proposed $1^{\text {st }}$ order Gaussian derivative FIR filter.

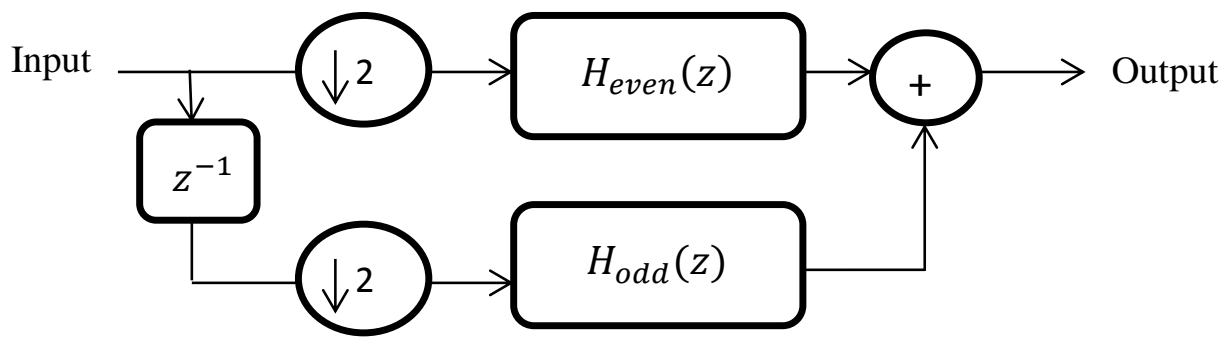

Fig. 6 Polyphase realization of the proposed FIR filter.

Using equation (1), the $1^{\text {st }}$ order Gaussian derivative filter can be derived with the coefficients values given in Table 1 .

Table 1: The values of coefficients of the $1^{\text {st }}$ order Gaussian derivative filter with $\sigma=4$

\begin{tabular}{|c|c|c|c|c|c|c|c|c|}
\hline Coefficient & $h_{o}=-h_{32}$ & $h_{1}=-h_{31}$ & $h_{2}=-h_{30}$ & $h_{3}=-h_{29}$ & $h_{4}=-h_{28}$ & $h_{5}=-h_{27}$ & $h_{6}=-h_{26}$ & $h_{7}=-h_{25}$ \\
\hline The value & $2.36^{*} 10^{-5}$ & $5.77 * 10^{-5}$ & $1.26 * 10^{-4}$ & $2.45^{*} 10^{-4}$ & $4.47 * 10^{-4}$ & $5.55^{*} 10^{-4}$ & $6.66 * 10^{-4}$ & $7.71 * 10^{-4}$ \\
\hline Coefficient & $h_{8}=-h_{24}$ & $h_{9}=-h_{23}$ & $h_{10}=-h_{22}$ & $h_{11}=-h_{21}$ & $h_{12}=-h_{20}$ & $h_{13}=-h_{19}$ & $h_{14}=-h_{18}$ & $h_{15}=-h_{17}$ \\
\hline The value & $8.61 * 10^{-4}$ & $9.22 * 10^{-4}$ & $9.45 * 10^{-4}$ & $9.19 * 10^{-4}$ & $8.41 * 10^{-4}$ & $7.08 * 10^{-4}$ & $4.74 * 10^{-4}$ & $2.46 * 10^{-4}$ \\
\hline Coefficient & $h_{16}$ & & & & & & & \\
\hline The value & 0 & & & & & & & \\
\hline
\end{tabular}

The values of the coefficients in Table 1 can be scaled (by $\alpha$ ) to give a maximum frequency response value that equals one, for the case of no-energy level variation during transformation. The value of $\alpha$ for $\left|H\left(e^{j w}\right)\right| \leq 1$ turns to be 70.795 . Therefore, the new scaled coefficients values are given in Table 2 .

Table 2: The scaled coefficient values of the $1^{\text {st }}$ order Gaussian derivative filter.

\begin{tabular}{|c|c|c|c|c|c|c|c|c|}
\hline Coefficient & $h_{o}=-h_{32}$ & $h_{1}=-h_{31}$ & $h_{2}=-h_{30}$ & $h_{3}=-h_{29}$ & $h_{4}=-h_{28}$ & $h_{5}=-h_{27}$ & $h_{6}=-h_{26}$ & $h_{7}=-h_{25}$ \\
\hline The value & 0.0017 & 0.0041 & 0.0089 & 0.0174 & 0.0317 & 0.0393 & 0.0471 & 0.0546 \\
\hline Coefficient & $h_{8}=-h_{24}$ & $h_{9}=-h_{23}$ & $h_{10}=-h_{22}$ & $h_{11}=-h_{21}$ & $h_{12}=-h_{20}$ & $h_{13}=-h_{19}$ & $h_{14}=-h_{18}$ & $h_{15}=-h_{17}$ \\
\hline The value & 0.0609 & 0.0653 & 0.0669 & 0.0651 & 0.0595 & 0.0501 & 0.0335 & 0.0174 \\
\hline Coefficient & $h_{16}$ & & & & & & & \\
\hline The value & 0 & & & & & & & \\
\hline
\end{tabular}


The corresponding magnitude and phase responses of $H(z)$ filter are shown in Fig. 7. Such figure indicates that the resulting filter is a band pass at low frequencies with linear phase.

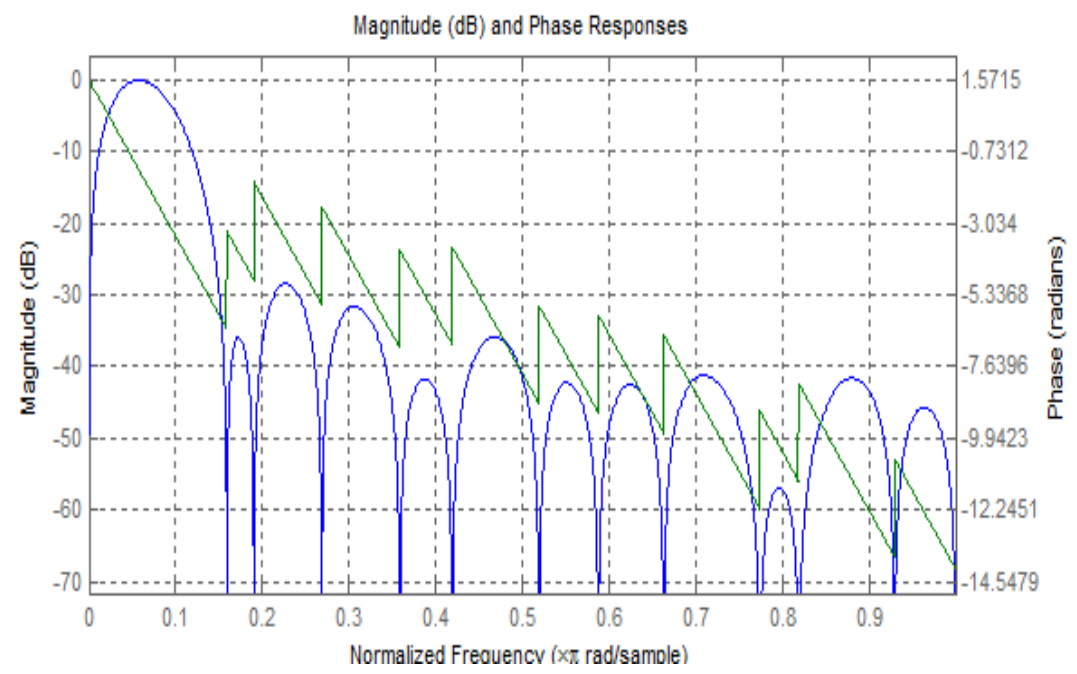

Fig. 7 Magnitude and phase responses of $H(z)$ filter.

The output of the designed FIR filter is the feature vectors of the original intervals of ECG signal, and then five standard measures parameters are extracted from these features diseases that used for classify diseases. These parameters are: energy of the feature vector that found according to eqn. (8), mean value of the feature vector as in eqn. (9), standard deviation of the feature vector as in eqn. (10), maximum of the feature vector and minimum of the feature vector are functions written in matlab program and used as in eqns.(11) \& (12), respectively. These parameters provide meaningful information for the diagnosis of possible heart. The obtained parameters will be defined as inputs to the classification system as in Fig. 8 .

$$
\begin{aligned}
& \text { Energy }=\sum_{n=1}^{N}\left|V^{2}\right| \\
& \text { Mean }=\frac{1}{N} \sum_{n=1}^{N} V
\end{aligned}
$$

maximum $=\max (V)$

where: $V$ : is the feature vector (filter output of Fig. 6) and $N$ : length of the feature.

\section{Classification Using Neuro-Fuzzy System}

Neuro-Fuzzy system with a Takagi-Sugeno Fuzzy inference system is implemented with five layered architecture as shown in Fig. 9. These layers are defined as the input linguistic layer (layer 1) that used for fuzzification of the input variables, Rule layer (layer 2), 
Normalization layer (layer 3), Defuzzification layer (layer 4) and the output linguistic layer (layer 5) that computes the overall inputs as the summation of all incoming signals [13].

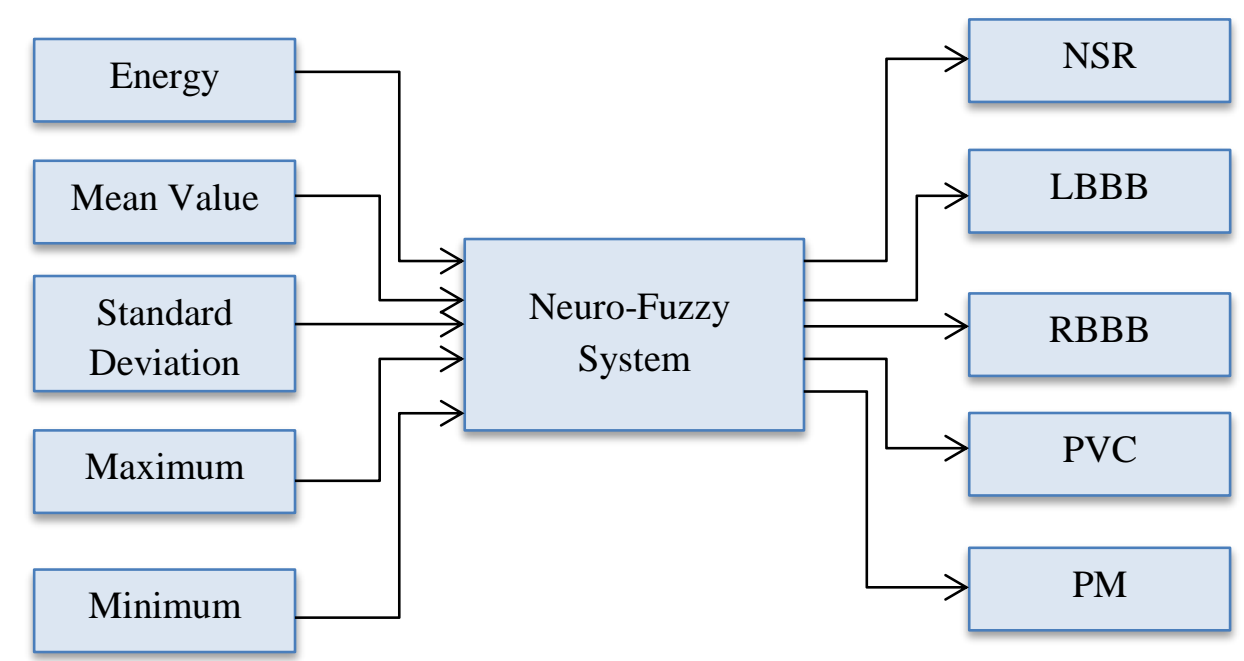

Fig. 8 Block diagram of heart disease classification using neuro-fuzzy system.

The nodes functions of neuro-fuzzy system in the same layer are described below [14].

Layer 1: all the nodes are adaptive nodes. The outputs of layer 1 are the fuzzy membership grade of the inputs, which are given by

$O^{1}{ }_{j}=\mu_{A_{j}{ }_{j}}\left(x_{i}\right)$

where $i=1,2, \ldots, n, j=1,2, \ldots, K, x_{\mathrm{i}}$ is the input to node $i, \mu_{A_{j}{ }_{j}}\left(x_{i}\right)$ denotes membership values of the $i^{\text {th }}$ input of the $j^{\text {th }}$ rule, where $A_{j}^{i}$ corresponds to a partition of the space of $x_{i}$

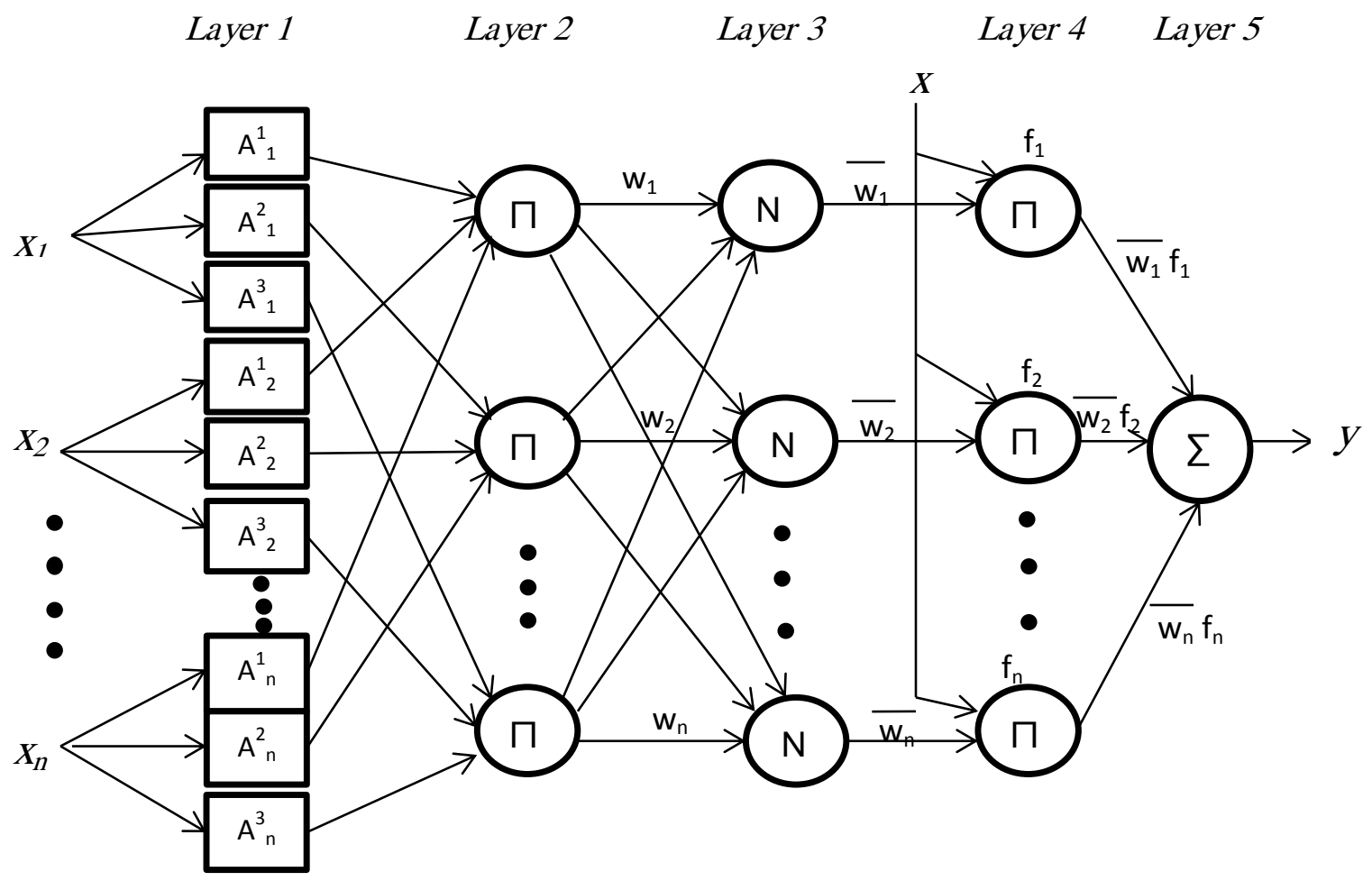

Fig. 9 Structure of the adopted neuro-fuzzy system. 
There are different shapes for the fuzzy membership functions such as Bell-shaped, Triangular, Gaussian, ...etc. $\mu_{A^{i}}{ }_{j}\left(x_{i}\right)$ is chosen to be a generalized Gaussian with maximum equal to 1 and minimum equal to 0 , having the following expression:

$\mu_{A^{j}{ }_{i}}\left(x_{i}\right)=e^{-\frac{1}{2}\left(\frac{x_{i}-c^{j} i}{a^{j} i}\right)^{2}}$

where $a^{j}{ }_{i}$ and $c^{j}{ }_{i}$ are the parameters set of the membership function. Parameters in this layer are referred to as "premise parameters".

Layer 2: every node in this layer is a fixed node labeled as $\prod$, whose output is the product of all incoming signals. The output of this layer can be represented as

$O^{2}{ }_{j}=w_{j}=\prod_{i=1}^{n} \mu A^{i}{ }_{j}\left(x_{i}\right) \quad$ for $i=1,2, \ldots, n \quad j=1,2, \ldots, K$

which are the so-called firing strengths of the rules.

Layer 3: every node in this layer is a fixed node labeled $N$. the $i^{\text {th }}$ nodes calculates the ratio of the rule's firing strength to the sum of all rules' firing strengths as represented by

$O_{j}^{3}=\overline{w_{J}}=\frac{w_{j}}{\sum_{j=1}^{K} w_{j}} \quad$ for $j=1,2, \ldots, K$

Outputs of this layer are called "normalized firing strengths"

Layer 4: the nodes in this layer are adaptive nodes. The output of each node in this layer is simply the product of the normalized firing strength and a first order polynomial (for a first order Sugeno model). Thus, the outputs of this layer are given by

$$
O^{4}{ }_{j}=\bar{w}_{J} f_{j}(x)
$$

where parameters in $f_{j}(x)$ are referred to as consequent parameters.

Layer 5: the single node in this layer is a fixed node labeled $\sum$ that computes the overall outputs as the summation of all incoming signals. Hence, the overall output of the model is given by

$$
O^{5}{ }_{j}=\sum_{j=1}^{K} \bar{w}_{J} f_{j}(x)=\frac{\sum_{j=1}^{K} w_{j} f_{j}(x)}{\sum_{j=1}^{K} w_{j}}
$$

Thus the functionally of the constructed adaptive network is equivalent to the TakagiSugeno type fuzzy inference system [8].

The graphical interfaces of the Takagi-Sugeno fuzzy rules and the values of membership functions that represent the rules regions created in rule based layer are shown in Fig. 10. The membership functions for input variables are shown in Fig. 11. 
Abdul-Jabbar: Neuro-Fuzzy Based ECG Signal Classification with A Gaussian-------
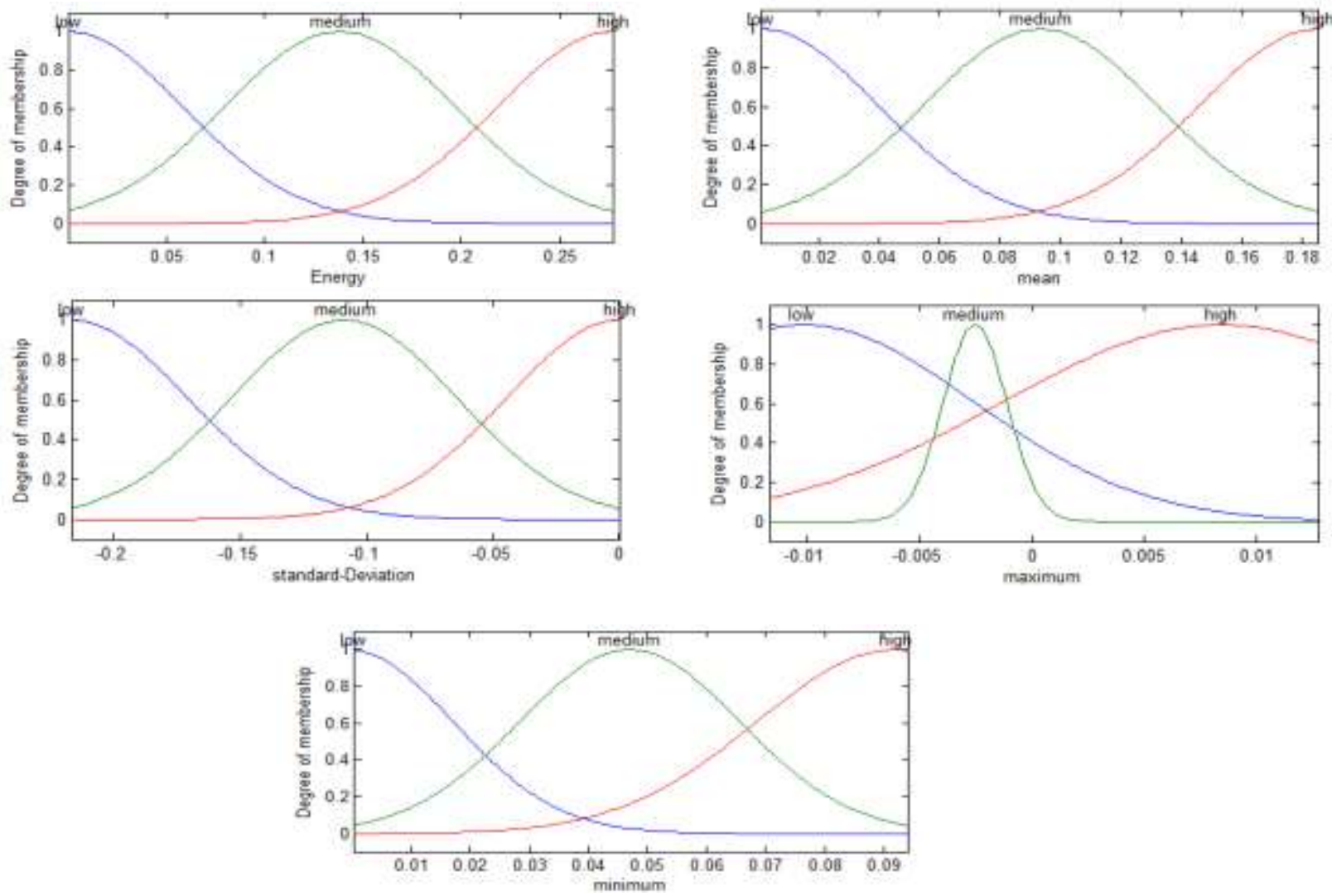

Fig. 10 Fuzzy rule base for the training set.

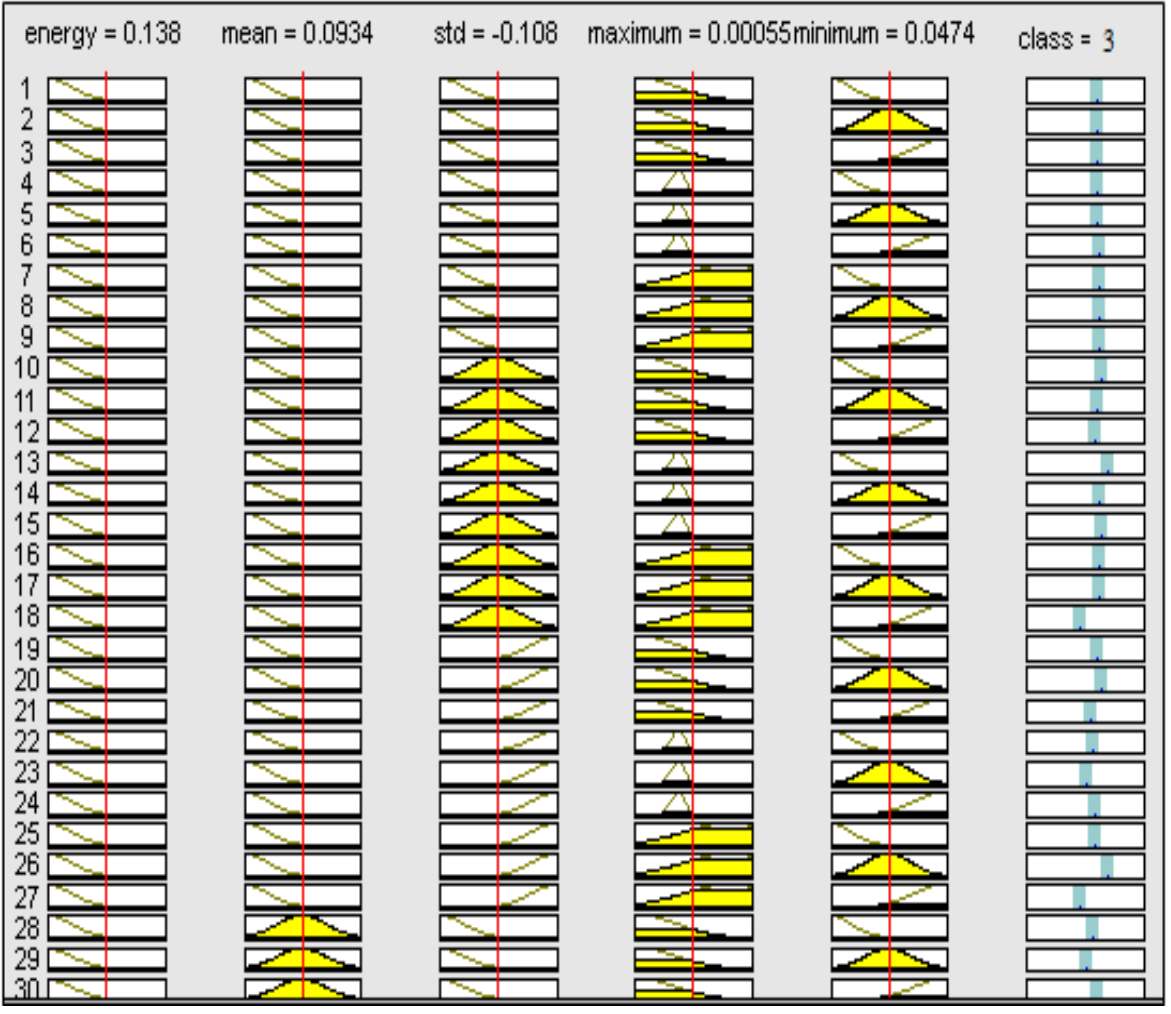

Fig. 11 Membership functions for different input variables. 


\section{RESULTS AND DISCUSSION}

The proposed classifier is implemented using the MIT-BIH arrhythmia database. A total of 450 datasets for five classes are adopted; out of which 250 datasets are used for training and the other 200 datasets are used for testing.

After training association rules in the form of If- Then rules are generated and extracted. An example of initial and final (after rule extraction) decision surfaces are given in Fig. 12. The classification results of the proposed Neuro-Fuzzy system (after 50 training set and 40 testing set) are shown in Table 3.

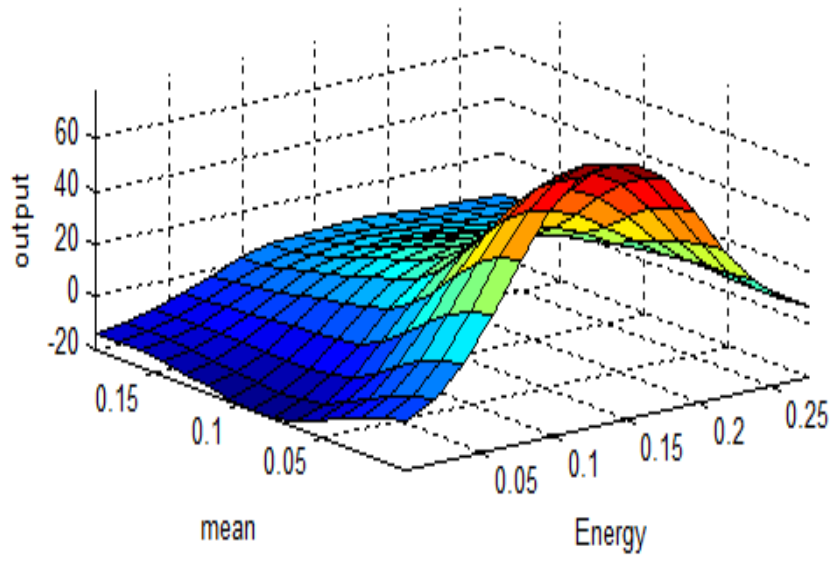

Fig. 12 Final surface for mean and Energy.

Table 3: Results of the proposed system.

\begin{tabular}{|c|c|c|c|c|}
\hline class & $\begin{array}{c}\text { Training } \\
\text { set }\end{array}$ & $\begin{array}{c}\text { Testing } \\
\text { set }\end{array}$ & $\begin{array}{c}\text { Correct } \\
\text { classified }\end{array}$ & $\begin{array}{c}\text { Mis- } \\
\text { classified }\end{array}$ \\
\hline NSR & 50 & 40 & 40 & 0 \\
\hline LBBB & 50 & 40 & 39 & 1 \\
\hline RBBB & 50 & 40 & 40 & 0 \\
\hline $\mathrm{PVC}$ & 50 & 40 & 40 & 0 \\
\hline PM & 50 & 40 & 39 & 1 \\
\hline \multicolumn{3}{|c|}{ Total } & 198 & 2 \\
\hline
\end{tabular}

From Table 3, it can be seen that a single left bundle branch block out of fifty is classified incorrectly as right bundle branch block and another one pacemaker is classified incorrectly as left bundle branch block.

\section{A. Performance Analysis}

The classification performance of the proposed Neuro-Fuzzy system is determined by the computation of statistical parameters such as sensitivity, specificity and accuracy. These parameters are defined in the following three equations [8]:

$$
\begin{aligned}
& \text { Sensitivity }=\frac{\text { No. of correct classified class }}{\text { Total no. of class signals }} * 100 \% \\
& \text { Specificity }=\frac{\text { No. of correct classified heart disease }}{\text { Total no. of heart disease }} * 100 \%
\end{aligned}
$$

$$
\text { Accuracy }=\frac{\text { Total no. of correct classification }}{\text { Total no. of testing signals }} * 100 \%
$$

The sensitivity, specificity for each ECG signal type with the overall accuracy for the proposed system are shown in Table 4 . The mean square error obtained for the proposed 
Neuro-Fuzzy system is 0.015. From Table 4, the neuro-fuzzy system showed significant results of the accuracy of the classification which are above $95 \%$. The accuracy rates presented are highly encouraging and suggest that neuro-fuzzy system is feasible in heart disease detection. That means, the proposed neuro-fuzzy classification system is an excellent system for predicting and classifying ECG signal. It is able to classify the extracted data from the first order Gaussian derivative filter of patients ECG signals efficiently and the neurofuzzy system can improve the classification quality for any ECG signal analysis application. Thus, it can help in improving the life of heart disease patient.

Table 4: Neuro-Fuzzy classification performance.

\begin{tabular}{|c|c|c|c|}
\hline \multirow{2}{*}{$\begin{array}{c}\text { ECG signal } \\
\text { types }\end{array}$} & \multicolumn{3}{|c|}{ statistical parameters } \\
\cline { 2 - 3 } & $\begin{array}{c}\text { Sensitivity } \\
\mathbf{\%}\end{array}$ & $\begin{array}{c}\text { Specificity } \\
\mathbf{\%}\end{array}$ & $\begin{array}{c}\text { Accuracy } \\
\mathbf{\%}\end{array}$ \\
\hline NSR & 100 & 100 & \\
\hline LBBB & 97.5 & 99 & \multirow{2}{*}{99} \\
\hline RBBB & 100 & 100 & \\
\hline PVC & 100 & 100 & \\
\hline PM & 97.5 & 99 & \\
\hline
\end{tabular}

Table 5: Results comparison of different methods.

\begin{tabular}{|c|c|c|}
\hline Method & Adopted Tools & Accuracy \\
\hline$[6]$ & Neuro-Fuzzy & $94 \%$ \\
\hline$[7]$ & ICA, Neuro-Fuzzy & $97.1 \%$ \\
\hline$[8]$ & DWT, Neuro-Fuzzy & $97.68 \%$ \\
\hline$[9]$ & PCA, NN & $97 \%$ \\
\hline $\begin{array}{c}\text { Proposed } \\
\text { method }\end{array}$ & $\begin{array}{c}\text { Gaussian derivative } \\
\text { filter, Neuro-Fuzzy }\end{array}$ & $99 \%$ \\
\hline
\end{tabular}

\section{B. A Comparative Study}

Table 5 summarizes the accuracy comparative results of the proposed method with some other recent different methods given in Refs. [6], [7], [8] and [9]. Such table indicates that the proposed method gives the best rate of accuracy.

\section{CONCLUSIONS}

In this paper, an ECG signal classification system has been proposed with the application of MIT/BIH arrhythmia data base. The algorithm consists of Sugeno fuzzy classifier and Backpropagation gradient descent method in combination with the least square method neural network.

The first Gaussian derivative filter has been used to extract features from the ECG diseases by applying a double-branch polyphase FIR filter. The recognition results of NSR, RBBB and PVC are better than other classes. A high classification accuracy of about $99 \%$ has been obtained using the proposed method, highlighting that this method can outperform many of the recent ones. 


\section{REFERENCES}

[1] L. K. Wee, Y. K. Jiar and E. Supriyanto, "Electrocardiogram Data Capturing System and Computerized Digitization using Image Processing Techniques", International Journal of Biology and Biomedical Engineering, Malaysia, Vol. 3, 2009, pp. 27-34.

[2] M. Engin," ECG Beat Classification using Neuro-Fuzzy Network", since direct Pattern Recognition Letters Elsevier, Electrical and Electronics Engineering Department, Faculty of Engineering, Ege University, Turkey, Vol. 25, 2004, pp. 1715-1722.

[3] S. J. Abou-Loukh, T. Zeyad and R. Thabit, "ECG Classification using Slantlet Transform and Artificial Neural Network", Journal of Engineering, Electrical Eng. Dept., College of Engineering, University of Baghdad, Iraq, Vol. 16, No. 1, 2010, pp. 4510-4528.

[4] V. Sadasivam, S. Bharathi and R.S. Kumari, "Design of Optimal Discrete Wavelet for ECG Signal using Orthogonal Filter Bank", IEEE International Conference on Computational Intelligence and Multimedia Applications,Vol.1, 2007, PP. 525-529.

[5] E. Kantoch, J. Jaworek and P. Augustyniak, "Design of a Wearable Sensor Network for Home Monitoring System", IEEE Federated Conference on Computer Science and Information Systems, 18-21 Sept. 2011, Poland, 2011, pp. 401-403.

[6] B. Anuradha, K. S. Kumar and V. C. Veera Reddy, "Classification of Cardiac Signals using Time Domain Methods", ARPN Journal of Engineering and Applied Sciences, Tirupati, India, No. 3, Vol. 3, June 2008, pp.7-12.

[7] T. M. Nazmy, H. EL-Messiry, and B. AL-Bokhity, "Adaptive Neuro-Fuzzy Inference System for Classification Of ECG Signals", Journal of Theoretical and Applied Information Technology, Faculty of computer and information sciences, Ain Shams University, Cairo, Egypt, Vol. 12, No. 2, 2010, pp. 71-76.

[8] N. H. Kamarudin, "Feature Extraction and Classification of Electrocardiogram Signal to Detect Arrhythmia and Ischemia Disease", M. Sc. Thesis, Department of computer science and information technology, University of Malaya, 2010.

[9] A. Sharma and T. Sharma, "ECG Beat Recognition using Principal Components Analysis and Artificial Neural Network ", International Journal of Electronics Engineering, Department of Electronics Engineering, University College of Eng., India, Vol. 1, No. 3, 2011, pp. 55-58.

[10] H. Gothwal, S. Kedawat and R. Kumar, "Cardiac Arrhythmias Detection In an ECG Beat Signal using Fast Fourier Transform and Artificial Neural Network", Journal Biomedical Science and Engineering, Department of Computer Science and Engineering, Malaviya National Institute of Technology, India, Vol. 4, 2011, pp. 289-296.

[11] N. A. Boon, N. R. Colledge and B. R. Walker, "Davidson Principle and Practical of Medicine", Edinburgh, Churchill Livingstone Elsevier, ISBN 0443100578, $20^{\text {th }}$ Edition, 2006, pp. 1126-1128.

[12] D. Hale, "Recursive Gaussian Filters", Center for Wave Phenomena, Colorado School of Mines, USA. On:

http://www.cwp.mines.edu/Meetings/Project06/cwp546.pdf.

[13] V. K. Srivastava and D. Prasad, "DWT - Based Feature Extraction from ECG Signal", American Journal of Engineering Research (AJER), Dept. of Electronics \& Comm. Eng., JJT University, Jhunjhunu, Rajasthan, India, Vol. 2, No. 3, 2013, pp. 44-50.

[14] Y. Wu, B. Zhang, J. Lu and K. -L Du, "Fuzzy Logic and Neuro-fuzzy Systems: A Systematic Introduction", International Journal of Artificial Intelligence and Expert Systems (IJAE), China, Vol. 2 , No. 2, 2011, pp.47-80. 